\title{
Comments on Kirkby and Polgar's interpretation of caudate nucleus function
}

\author{
GORDON WINOCUR \\ Trent University, Peterborough, Ontario, Canada
}

\begin{abstract}
Kirkby and Polgar's position that the antero-dorsal caudate nucleus is important for two-way avoidance conditioning ( $\mathrm{AC}$ ) is supported by recent experiments, but their argument against differential involvement of dorsal and ventral caudate regions in one-way AC is less substantiated. Kirkby's unitary explanation of caudate nucleus function in terms of arousal control does not incorporate important aspects of the caudate deficit pattern and fails to take into account the considerable functional heterogeneity within that structure.
\end{abstract}

In a series of experiments, Kirkby and Polgar (1974) compared the effects of lesions to dorsal or ventral aspects of the head of the caudate nucleus on performance in one- and two-way active avoidance conditioning (AC) tasks. With one exception, their results are identical to those of similar experiments previously published by Winocur (1974). The major difference is that Kirkoy and Polgar found dorsal and ventral groups to be equally impaired relative to controls on one-way $\mathrm{AC}$ while Winocur found only ventral groups to be impaired on this task. The ability of rats with dorsal caudate lesions to learn a one-way avoidance response within normal limits had also been previously reported by Winocur and Mills (1969) who argued that the antero-dorsal aspect of the caudate nucleus was not essential for active AC. Kirkby and Polgar (1974) maintain that this hypothesis should be rejected.

Insofar as the Winocur and Mills hypothesis referred to two-way, as well as one-way AC, Kirkby and Polgar's point has justification. Until recently, only one experiment (Albert \& Bignami, 1968) had appeared in which rats with restricted antero-dorsal caudate lesions had been tested on shuttlebox AC. These animals performed as well as controls but subsequent experiments by Neill and Grossman (1970), Winocur (1974), and most recently by Kirkby and Polgar (1974), involving essentially the same task have produced data contradictory to the results of Albert and Bignami. Lesions placed more ventrally in the structure will also impair two-way AC (Mitcham \& Thomas, 1972), but it should be emphasized that the respective contributions of antero-dorsal and postero-ventral caudate regions are quite different (Winocur, 1974).

Kirkby and Polgar also argue against the notion that dorsal and ventral regions of the caudate nucleus are differentially involved in one-way AC. In this respect, their position is more difficult to accept. Only two published studies have directly compared dorsal and ventral caudate involvement in one-way AC (Kirkby \& Polgar, 1974; Winocur, 1974), and they are clearly contradictory regarding the performance of the dorsal groups. However, Winocur and Mills (1969) had earlier tested different groups of rats with restricted antero-dorsal caudate lesions under three conditions of one-way $\mathrm{AC}$ and consistently found no significant differences between these groups and controls. On the other hand, the results of a number of studies (e.g., Kirkby \& Kimble, 1968; Kirkby \& Polgar, 1974; Winocur, 1974) leave little doubt that caudate lesions involving postero-ventral regions severely disrupt one-way avoidance conditioning. Thus, taken together, the bulk of the evidence suggests that the caudate nucleus is functionally dissociated with respect to $\mathrm{AC}$ and that antero-dorsal portions of the structure are not essential for one-way learning. The functional heterogeneity of the rat caudate nucleus has been extended to passive as well as active avoidance learning (Winocur, 1974), appetitive discrimination tasks (Potegal, 1969), and to a variety of tasks involving other species (see review by Divac, 1968).

Kirkby (1973; see also Kirkby \& Polgar, 1974) favors a unitary explanation of caudate function. In a series of experiments, Kirkby (1973) found rats with caudate lesions [larger than those in the Winocur $(1969,1974)$ and Kirkby \& Polgar (1974) studies] to be hyperreactive in a variety of test situations including, for example, open field behavior and reactivity to illumination. His interpretation that the caudate nucleus plays a role in "the modulation of neural arousal" (p. 94) conforms to his data but is limited as a general statement of caudate function. For example, an extremely aroused animal with low response thresholds could be expected to exhibit increased motor excitability in a variety of situations, particularly those demanding response suppression. Although rats with caudate damage are sometimes impaired on tasks which involve the withholding of inappropriate responses, e.g., passive avoidance conditioning, this impairment seems related to the effects of the lesion interacting with specific task conditions (Winocur \& Mills, 1969) rather than a lesion-induced bias towards response disinhibition. Furthermore, rats rendered hyperreactive by brain 
lesions, as is the case following damage to the septal region, typically show enhanced two-way AC (Krieckhaus, Simmons, Thomas, \& Kenyon, 1964; Schwartzbaum, Green, Beatty, \& Thomson, 1967). As already established, caudate lesions severely impair this type of learning.

An experiment by Schmaltz and Isaacson (1968) may explain in part the increased activity pattern attributed to rats with caudate nucleus lesions. These investigators found that, following caudate lesions, rats displayed abnormally high response rates and consequently achieved little success in a DRL barpressing task. The effect, however, was transient so that by $40-45$ days postsurgery, response differences between groups had disappeared. In this context, it is significant that in Kirkby's (1973) experiments, all testing was completed within 28 days of surgery. The possibility that caudate-damaged rats are sometimes slower to recover from the irritative effects of the lesions should at least be considered.

Thus, Kirkby's (1973) explanation of caudate function does not adequately incorporate many important aspects of the caudate deficit pattern and if application is restricted to transient postoperative changes, its ability to describe the behavioral role of the structure in any fundamertal sense becomes questionable. Finally, and perhaps most importantly, the hypothesis does not take into account the well-established functional heterogeneity of the caudate nucleus, one advantage at least of hypotheses favored by other investigators (Divac, 1968; Potegal, 1969; Winocur, 1974).

\section{REFERENCES}

Albert, M., \& Bignami, G. Effect of frontal, median cortical and caudate lesions on two-way avoidance learning by rats. Physiology and Behavior, 1968, 3, 141-147.

Divac, I. Functions of the caudate nucleus. Acta Biologiae Experimentalis, Warsaw, 1968, 28, 107-120.

Kirkby, R. J. The caudate nucleus and arousal in the rat. Journal of Comparative and Physiological Psychology, 1973, 85, 82-96.

Kirkby, R. J., \& Kimble, D. P. Avoidance and escape behavior following striatal lesions in the rat. Experimental Neurology, $1968,20,215-227$.

Kirkby, R. J., \& Polgar, S. Active avoidance in the laboratory rat following lesions of the dorsal or ventral caudate nucleus. Phy siological Psy chology, 1974, 2, 301-306.

Krieckhaus, E. E., Simmons, H. J., Thomas, G. J., \& Keny on, J. Septal lesions enhance shock avoidance behavior in the rat. Experimental Neurology, 1964, 9,107-113.

Mitcham, J. C., \& Thomas, R. K., Jr. Effects of substantia negra and caudate nucleus lesions on avoidance learning in rats. Joumal of Comparative and Physiological Psychology, 1972, 81, 101-107.

Neill, D. B., \& Grossman, S. P. Behavioral effects of lesions on cholinergic blockade of the dorsal and ventral caudate of rats. Journal of Comparative and Physiological Psychology, 1970, 71, 311-317.

Potegal, M. Role of the caudate nucleus in spatial orientation of rats. Journal of Comparative and Physiological Psychology. $1969,69,756-764$.

Schmaltz, L. W., \& Isaacson, R. L. Effects of caudate and frontal lesions on retention and relearning of a DRL schedule. Journal of Comparative and Physiological Psychology, 1968, 65. 343-348.

Schwartzbaum, J. S., Green, R. H., Beatty, W. W., \& Thompson, G. B. Acquisition of avoidance behavior following septal lesions in the rat. Joumal of Comparative and $\mathrm{Phy}$ siological Psy chology, 1967, 63, 95-104.

Winocur, G. Functional dissociation within the caudate nucleus of rats. Joumal of Comparative and Phy siological Psychology, $1974,86,432-439$.

Winocur, G., \& Mills, J. A. Effects of caudate lesions on avoidance behavior in the rat. Joumal of Comparative and Physiological Psy chology, 1969, 68, 552-557.

(Received for publication December 30, 1974; accepted February 1, 1975.) 